\title{
Una propuesta piloto de herramienta analítica del aprendizaje para la mejora de procesos colaborativos en la plataforma Blackboard
}

\author{
Shamaly Alhelí Niño Carrasco (D) @ \\ Juan Carlos Castellanos Ramírez (i) @ \\ Esperanza Viloria Hernández (D) @ \\ ${ }^{1}$ Universidad Autónoma de Baja California (UABC), México
}

Resumen. Se presenta un estudio centrado en un conjunto de indicadores relacionados con el modelo de influencia educativa distribuida, susceptibles de desarrollar una herramienta analítica del aprendizaje, que ayude a proporcionar feedbacks formativos. Para ello, se explora el efecto que la entrega de información relacionada con este modelo tiene sobre la mejora de los procesos colaborativos de los participantes en la plataforma digital Blackboard. Se realizó un estudio cualitativo de casos con 24 estudiantes de licenciatura, que participaron en cinco foros de discusión en línea. Los resultados muestran que la entrega de información, potencia la mejora de los procesos colaborativos en las etapas iniciales de la tarea. Se concluye, que la información asociada al modelo teórico tiene un efecto mayor sobre los procesos colaborativos de los grupos que contaron con esta información; esto refuerza el interés por analizar, en profundidad, las dinámicas particulares de cada grupo para comprender el efecto de la información en las mismas.

Palabras clave: aprendizaje colaborativo; aprendizaje virtual; construcción del conocimiento; educación superior; tecnología digital.

Uma proposta piloto de ferramenta analítica de aprendizagem para a melhoria de
processos colaborativos na plataforma Blackboard
Resumo. Apresenta-se um estudo centrado em um conjunto de indicadores relativos ao
modelo de influência educacional distribuída e que são capazes de desenvolver uma
ferramenta analítica de aprendizagem, facilitando o fornecimento de feedbacks para
a formação. Portanto, é analisado o efeito que a entrega das informações relacionadas
com este modelo tem sobre a melhoria dos processos colaborativos dos participantes
da plataforma digital Blackboard. Um estudo de caso qualitativo foi realizado com 24
estudantes de graduação, que participaram de cinco fóruns de discussão on-line.
Os resultados mostram que a entrega de informação potencializa a melhoria dos
processos colaborativos nos estágios iniciais da tarefa. Conclui-se que as informações
associadas ao modelo teórico têm um efeito maior sobre os processos colaborativos
dos grupos que obtiveram estas informações. Isso reforça o interesse em analisar mais
detalhadamente a dinâmica particular de cada grupo, a fim de compreender o efeito
da informação em cada um deles.

Palavras-chave: aprendizagem colaborativa; aprendizagem virtual; construção do conhecimento; educação superior; tecnologia digital.

Developing a pilot Learning Analytic tool for the improvement of collaborative processes in Blackboard platform.

Abstract. This work presents a study focused on a set of indicators related to the model of distributed educational influence that helps the design of a Learning Analytic to provide formative feedback. It explores the effect of providing information related to such model on the collaborative processes of the participants in Blackboard digital environment. $A$ qualitative case study was conducted with 24 undergraduate students who participated in five online discussion forums. The results suggest that providing information has an effect on the improvement of collaborative processes just at the initial phases. It is concluded that the information related to the theoretical model has a greater effect on the collaborative processes of the groups that handled this information; the above idea reinforces the interest to analyze, in depth, the particular dynamics of each group to understand the effect of the information on the configuration of such dynamics.

Keywords: collaborative learning; digital technology; higter education; knowledge construction; virtual learning. 


\section{Introducción}

El uso de las tecnologías digitales para apoyar y guiar los procesos colaborativos de los estudiantes es en la actualidad uno de los focos que más interés despierta en la investigación psicoeducativa (Dillenbourg, Järvelä, y Fischer, 2009; Kirschner y Erkens, 2013; Jeong y Hmelo-Silver, 2016). En este ámbito ocupa un lugar destacado el campo de investigación y diseño conocido como analíticas del aprendizaje (LA, por sus siglas en inglés), definido como "the measurement, collection, analysis and reporting of data about learners and their contexts, for purposes of understanding and optimizing learning and the environments in which it occurs" (Siemens y Long, 2011, p. 34).

Otras propuestas cercanas como las herramientas del análisis de la interacción (Dimitracopoulou, 2008), de conciencia de grupo (Dehler, Bodemer, Buder y Hesse, 2011) o la minería de datos educativos (García, Romero, Ventura, y de Castro, 2011) comparten con las herramientas analíticas el interés en diseñar herramientas para proporcionar información a los participantes en tiempo real sobre la actividad que desarrollan, con el fin de ayudarlos a mejorar los procesos de colaboración. Estas líneas de investigación, más allá de las diferencias de estas propuestas, comparten el desarrollo de métodos para explotar el enorme caudal de datos sobre la actividad de los usuarios, que quedan registradas automáticamente en las plataformas educativas, con el fin de optimizar los procesos de enseñanza y aprendizaje.

La finalidad última, es que la información brindada a los profesores y estudiantes guíe y oriente sus actuaciones, ayudándoles a mejorar sus experiencias de enseñanza y aprendizaje en línea. No obstante, algunos autores (Brown, 2012; Ferguson, 2012) destacan que el desarrollo de una herramienta analítica que contribuya a mejorar el aprendizaje colaborativo en línea, requiere fundamentar teóricamente los datos que deben recopilarse, su procesamiento e, incluso, los formatos de presentación y visualización. En la misma línea, Durall y Gros (2014) y Gros (2016) señalan la importancia de que las herramientas analíticas de aprendizaje constituyan una fuente de apoyo para generar feedbacks formativos, que sean útiles para profesores y estudiantes.

En este marco, el trabajo que ahora se presenta es una propuesta piloto de herramienta analítica del aprendizaje, sustentada en un conjunto de indicadores basados en el modelo de influencia educativa distribuida (IED), que han sido desarrollados desde el ámbito de la psicología educativa (Coll, Bustos, y Engel, 2015; Coll, Engel, y Niño 2017). Por lo tanto, el objetivo de este trabajo es analizar el efecto que tiene proporcionar información basada en los indicadores de IED sobre los procesos colaborativos de los participantes en una plataforma digital Blackboard. 
Para ello se describe el modelo de IED y las dimensiones e indicadores que se han retomado desde este modelo para ser aplicados en la plataforma digital Blackboard. Después, se presenta el marco metodológico empleado para recopilar, analizar y entregar a los participantes la información de su propia actividad. Posteriormente, se presentan los resultados vinculados con el objetivo de la investigación y, finalmente, se valoran los resultados y las implicaciones para la mejora de la propuesta.

\section{El modelo de influencia educativa distribuida}

El concepto de ayuda o influencia educativa (IE) nace de una serie de trabajos sobre el análisis de las prácticas educativas en contextos presenciales, fundamentados en una aproximación constructivista y sociocultural de los procesos de enseñanza y aprendizaje (ver, por ejemplo, Coll, Colomina, Onrubia, y Rochera, 1992; Coll, Onrubia, y Mauri, 2008; Colomina, Onrubia, y Rochera, 2001). La IE hace referencia a los procesos interpsicológicos mediante los cuales los profesores, u otros agentes educativos, ayudan a los estudiantes a construir significados progresivamente más ricos, complejos y válidos sobre los contenidos de enseñanza y aprendizaje.

Sin embargo, no toda ayuda constituye necesariamente una ayuda eficaz. La condición básica que deben cumplir los procesos de ayuda o IE para ser eficaces, es la de estar estrechamente vinculados y ajustados al desarrollo y evolución de los procesos de construcción de significados y de atribución de sentido que Ilevan a cabo los estudiantes. Por lo tanto, esta ayuda tiene necesariamente que ser contingente y variada, en cantidad e intensidad, para ajustase a los avances y retrocesos que presenten los estudiantes a lo largo del proceso de aprendizaje (Coll, 2010).

Como se aprecia, en las situaciones de construcción guiada del conocimiento, la fuente principal de ayudas y apoyos al aprendizaje es el profesor. Sin embargo, en los procesos de construcción colaborativa del conocimiento, todos los participantes son fuentes potenciales de ayuda educativa para el resto (Bustos, 2011; Bustos, Coll, y Engel, 2009; Coll, Bustos, Engel, de Gispert, y Rochera, 2013). Partiendo de lo anterior, el concepto de influencia educativa distribuida (IED) subraya la idea de que el ejercicio de la IE en las situaciones colaborativas se distribuye entre todos los participantes, ya que todos son fuentes potenciales de ayuda y pueden, en mayor o menor medida, contribuir al desarrollo de procesos de aprendizaje más complejos. 
En concreto, en los procesos de aprendizaje colaborativo mediados por ordenador, la posibilidad de que los participantes sean capaces de ofrecer y recibir ayudas de manera efectiva depende del cumplimiento de ciertas exigencias (Coll, Bustos, y Engel, 2011; 2015; Coll, Engel, y Bustos, 2009; Engel, Coll, y Bustos, 2013). El primer tipo de exigencias, que los autores refieren, está relacionado con el acceso periódico que los participantes deben realizar al entorno digital, con realizar contribuciones y leer las publicaciones del resto de los participantes y, además, con el hecho de establecer relaciones recíprocas y responsivas a través de lo que se escriben y se responden.

El segundo tipo de exigencias remite mucho más al contenido y a la calidad de las contribuciones de los participantes, por lo que se espera que entre todos gestionen las tres dimensiones esenciales que, desde el marco teórico de IED, son esenciales para el desarrollo de los procesos de enseñanza y aprendizaje: la gestión de la participación social, de la tarea académica y de los significados. Como Coll, Engel y Niño (2017) comentan, la gestión de la participación social remite a quién puede hacer o decir qué, cuándo, cómo, dirigiéndose a quién y con qué medios; la gestión de la tarea académica versa sobre el qué hay que hacer, cómo y mediante qué procedimientos, qué tarea académica se debe generar y qué características debe tener; y la gestión de los significados está relacionada con los contenidos de enseñanza y aprendizaje, como son la presentación de información, las peticiones de aclaraciones, las manifestaciones de acuerdo, las valoraciones críticas sobre lo que se está construyendo, entre otras.

El modelo de IED (Coll, Engel, y Niño, 2017) contempla dos tipos de indicadores que, por un lado, informan sobre los aspectos y dimensiones de la actividad colaborativa e individual de los participantes y, por otro lado, constituyen una buena base para diseñar una herramienta analítica con las características que ya se han mencionado. De acuerdo con la tabla 1 , en el primer tipo de indicadores (celdas sombreadas) se encuentran los índices de carácter estructural relacionados con el primer grupo de exigencias que se mencionaron (acceso al entorno, escritura y lectura de contribuciones, establecimiento de relaciones); el segundo grupo de indicadores (celdas sin sombrear) está basado en el contenido de las contribuciones relacionados con las tres dimensiones de la gestión de la IED. 
Tabla 1. Indicadores de carácter estructural y de contenido asociados al modelo de IED

Indicadores Descripción

Índice individual de acceso Número de días que un participante accede al entorno en línea (IIA) en el que se desarrolla la actividad sobre el número total de días de duración de la actividad

Patrón individual de acceso Continuidad del acceso al entorno en línea (PIA)

Índice individual de lecturas Proporción de contribuciones leídas por un participante con (IIL) respecto al total de contribuciones hechas por el resto de los participantes

Índice individual de contri- Proporción de contribuciones escritas por un participante en buciones (IIC) relación con las requeridas para la realización de la tarea

Patrón individual de contri- Distribución de las contribuciones escritas por un participante a buciones (PIC) lo largo del desarrollo de la tarea

índice individual de recipro- Proporción de relaciones comunicativas recíprocas que establece cidad (IIR) un participante con otros participantes sobre el total de relaciones recíprocas posibles que podría llegar a establecer

Índice grupal de acceso (IGA) Sumatorio de los días que los diferentes participantes acceden al entorno en línea sobre los días de duración de la actividad por el número de participantes

Índice grupal de lecturas Total de contribuciones leídas por todos los participantes sobre (IGL) el número de lecturas posibles de las contribuciones realizadas por todos los participantes

Índice grupal de contribucio- Total de contribuciones escritas por todos los participantes sobre nes (IGC) el número esperado de contribuciones según las directrices de desarrollo de la actividad

Índice de densidad de la Proporción de relaciones comunicativas reales que establecen los red (IDR) participantes con otros participantes sobre el total de relaciones posibles que podrían llegar a establecer [n(n-1)]

Índicegrupal de reciprocidad Proporción de pares de participantes que tienen conexión recí(IGR) proca entre sí

índice individual de gestión Contribuciones o fragmentos de contribuciones que el participante de la participación social (IIP) dedica a gestionar la participación social sobre el total de contribuciones o fragmentos de contribuciones del mismo participante

Índice individual de gestión Contribuciones o fragmentos de contribuciones que el participante de la tarea académica (IIT) dedica a gestionar la tarea académica sobre el total de contribuciones o fragmentos de contribuciones del mismo participante

Índice individual de gestión Contribuciones o fragmentos de contribuciones que el participante de los significados (IIS) dedica a gestionar los significados sobre el total de contribuciones o fragmentos de contribuciones del mismo participante

índice grupal de gestión de Contribuciones ofragmentos de contribuciones que el grupo dedica la participación social (IGP) agestionar la participación social sobre el total de contribuciones o fragmentos de contribuciones del conjunto de miembros del grupo

índice grupal de gestión de la Contribuciones o fragmentos de contribuciones que el grupo dedica tarea académica (IGT) a gestionar la tarea académica sobre el total de contribuciones o fragmentos de contribuciones del conjunto de miembros del grupo

índice grupal de gestión de Contribuciones o fragmentos de contribuciones que el grupo delos significados (IGS) dica a gestionar los significados sobre el total de contribuciones 0 fragmentos de contribuciones del conjunto de miembros del grupo

Nota. Adaptada de Coll, Engel y Niño, 2017, p. 6-8. 
Para fines del estudio, del conjunto de índices presentados en la tabla anterior se han seleccionado únicamente aquellos que permiten un rápido análisis de la actividad de los participantes y, sobre todo, aquellos cuyos registros de actividad permitieron un acceso directo a los investigadores. Así, de los indicadores de tipo estructural se han seleccionado el IIA, el PIA, el IIC, el PIC y el IIR; de los indicadores derivados del contenido de las contribuciones, únicamente se han considerado los índices individuales que son el IIP, el IIT y el IIS.

Como se muestra, más adelante, el conjunto de valores obtenidos en estos índices e indicadores permite confeccionar el perfil de cada participante y clasificarlo en función de su proximidad o lejanía a un perfil teóricamente idóneo según el modelo de IED.

\section{Metodología}

El trabajo realizado puede caracterizarse como un estudio de caso en situación natural (Yin, 2006), aproximación metodológica utilizada habitualmente para analizar y comprender en profundidad procesos de aprendizaje colaborativo en línea (Stahl, Koschmann, y Suthers, 2006).

\subsection{Contexto y participantes}

Participaron 24 estudiantes (20 mujeres y cuatro hombres) que cursaban una asignatura de carácter obligatorio de una licenciatura en la Universidad Autónoma de Baja California; los contenidos de enseñanza y aprendizaje se abordaron a lo largo de dieciséis sesiones presenciales.

De dichos contenidos temáticos, el estudio comprende aquellas actividades que los estudiantes desarrollaron, paralelamente, en línea. En concreto, las situaciones de observación del estudio corresponden a los contenidos temáticos desarrollados en cinco semanas: en el periodo comprendido entre una sesión presencial y la siguiente, los 24 estudiantes del grupo clase organizados en cuatro grupos, formados al azar por 6 estudiantes cada uno, debían trabajar de manera colaborativa y asíncrona en la elaboración de un informe escrito que contemplara los rasgos distintivos del contenido temático abordado en la sesión presencial. Los estudiantes contaban para elaborar dicho informe con un foro de comunicación de la plataforma Blackboard. 


\subsection{Diseño}

Para valorar el efecto que la información de la actividad de los participantes tiene sobre sus propios procesos, al inicio de cada foro de elaboración del informe, se les entregó información individual sobre la actividad del foro que acababan de finalizar; para ello se utilizó el correo electrónico institucional de los participantes. Por lo tanto, de los cinco foros programados, los estudiantes recibieron la información correspondiente a la actividad desarrollada en cuatro foros: la primera entrega de información se realizó toda vez que concluyó el foro 1 con fines de mejora de la actividad del foro 2 , las tres entregas restantes también se realizaron al término de cada foro y previo al inicio del siguiente.

Cabe señalar que se establecieron cuatro condiciones distintas. Así, a un grupo de estudiantes -GA- se le proporcionó la información derivada del análisis estructural de su actividad. El conjunto de indicadores de este primer tipo de información se calculó con los datos recogidos directamente de la plataforma Blackboard, ya que al ser una plataforma que opera de manera institucional, no fue posible acceder a los registros de actividad del curso. La tabla 2 presenta los indicadores que se retomaron de la propuesta de Coll, Engel y Niño (2017) y para los que se señaló el valor por encima del cual se consideraba que el nivel de actividad del participante era teóricamente adecuado para ejercer la IE en el aspecto o dimensión al que remite el índice en esta actividad en particular (ver tabla 2).

Tabla 2. Información de naturaleza estructural entregada a los participantes del GA

\begin{tabular}{ll}
\hline \multicolumn{1}{c}{ Indicadores individuales } & \multicolumn{1}{c}{ Valor idóneo } \\
\hline Índice individual de acceso (IIA) & $\begin{array}{l}\geq 0,50 \text {; el participante accede, al menos, cuatro } \\
\text { días a la plataforma } \\
\text { Patrón individual de acceso (PIA) }\end{array}$ \\
$\begin{array}{l}\text { Continuo, cuando el participante no presenta } \\
\text { ningún período de más de dos días sin acceso } \\
\text { al foro en línea }\end{array}$ \\
Índice individual de contribuciones (IIC) & $\begin{array}{l}\geq 1,50 ; \text { el participante contribuye más de lo } \\
\text { requerido en la actividad } \\
\text { Índice individual de reciprocidad (IIR) }\end{array}$ \\
& $\begin{array}{l}\geq 0,60 \text {; el participante serelacionarecíprocamente } \\
\text { con la mayoría de los participantes }\end{array}$ \\
\hline
\end{tabular}

A otro grupo de estudiantes -GB- se le entregó información derivada del análisis de contenido de las contribuciones aportadas en el foro anterior. En este caso, la elaboración de la información se realizó mediante la codificación de las contribuciones de los participantes en términos de las tres dimensiones implicadas en el ejercicio de la IED. A diferencia de los 
indicadores anteriores, para los indicadores derivados del análisis de contenido de las contribuciones no se señalaba un nivel de actividad más adecuado que otro para ejercer la IE (ver la tabla 3).

Tabla 3. Información sobre el contenido de las contribuciones entregada a los participantes del GB

\begin{tabular}{|c|c|}
\hline Dimensiones & Indicadores \\
\hline $\begin{array}{l}\text { índice individual de ges- } \\
\text { tión de la participación } \\
\text { social (IIP) }\end{array}$ & $\begin{array}{l}\text { \% individual de las categorías de participación social identificadas } \\
\text { en las contribuciones e interpretación sobre cómo se realizó la } \\
\text { gestión de esta dimensión. }\end{array}$ \\
\hline $\begin{array}{l}\text { índice individual de ges- } \\
\text { tión de la tarea académica } \\
\text { (IIT) }\end{array}$ & $\begin{array}{l}\text { \% individual de las categorías de tarea académica identificadas en } \\
\text { las contribuciones e interpretación sobre cómo se realizó la gestión } \\
\text { de esta dimensión. }\end{array}$ \\
\hline $\begin{array}{l}\text { índice individual de ges- } \\
\text { tión de los significados } \\
\text { (IIS) }\end{array}$ & $\begin{array}{l}\text { \% individual de las categorías de significados identificadas en las } \\
\text { contribuciones e interpretación sobre cómo se realizó la gestión de } \\
\text { esta dimensión. }\end{array}$ \\
\hline
\end{tabular}

A un tercer grupo de estudiantes -GC- se entregó los dos tipos de informaciones anteriores. Finalmente, un cuarto grupo de participantes -GDrecibió únicamente valoraciones generales sobre el cumplimiento de la tarea.

\subsection{Análisis de los datos}

Como ya se adelantaba, para el análisis de la actividad de los participantes se han utilizado los datos registrados directamente de la plataforma Blackboard. El procedimiento seguido ha consistido en seleccionar el conjunto de indicadores de tipo estructural mencionados en la Tabla 2. El análisis realizado a través de dichos indicadores ha permitido identificar perfiles individuales de actividad que pueden ser valorados en función de su mayor o menor proximidad a un perfil teóricamente idóneo.

En la tabla 4 se presenta el número total de datos recogidos en cada foro para realizar el cálculo de los indicadores mencionados y la identificación de los perfiles de actividad.

De manera adicional, para analizar la utilidad que los participantes otorgan a la información entregada para mejorar su actividad en los foros, se les administró un cuestionario después de cada entrega. En total, se administraron cuatro cuestionarios en los que los participantes tuvieron que valorar el grado de utilidad de las informaciones recibidas, mediante una escala tipo Likert de cuatro puntos. A efectos de visualizar la evolución de las valoraciones sobre la información entregada se han sumado las puntuaciones obtenidas por cada ítem en cada foro. 
Tabla 4. Número total de datos recogidos en cada foro

\begin{tabular}{clrrrrr}
\hline Grupo & & F1 & F2 & F3 & F4 & F5 \\
\hline \multirow{2}{*}{ GA } & Días diferentes de acceso & 4 & 6 & 5 & 5 & 4 \\
& Contribuciones & 50 & 48 & 54 & 51 & 49 \\
& Relaciones recíprocas & 13 & 19 & 14 & 22 & 14 \\
\multirow{3}{*}{ GB } & Días diferentes de acceso & 3 & 4 & 4 & 5 & 4 \\
& Contribuciones & 59 & 80 & 72 & 61 & 63 \\
& Relaciones recíprocas & 14 & 19 & 17 & 19 & 20 \\
\multirow{3}{*}{ GC } & Días diferentes de acceso & 3 & 7 & 6 & 5 & 5 \\
& Contribuciones & 47 & 60 & 64 & 62 & 52 \\
& Relaciones recíprocas & 9 & 18 & 19 & 18 & 15 \\
& Días diferentes de acceso & 3 & 4 & 4 & 4 & 4 \\
& Contribuciones & 40 & 51 & 58 & 41 & 41 \\
& Relaciones recíprocas & 10 & 13 & 15 & 10 & 11 \\
\hline
\end{tabular}

\section{Resultados}

En este apartado se presentan, primero, los resultados correspondientes a la evolución de los indicadores relacionados con el ejercicio de la IE alcanzados por los grupos a lo largo del desarrollo de los distintos foros $\mathrm{y}$, después, los resultados relativos a las valoraciones que los participantes hacen sobre la utilidad de la información recibida.

\subsection{Evolución de los índices}

Como se aprecia en la tabla 5, hay sendas diferencias entre el número de participantes que alcanzan el valor idóneo de los cuatro indicadores en el F1 y el resto de los foros; sin embargo, el cumplimiento de cada indicador varía entre cada grupo. En detalle, en los cuatro grupos se observa que el IIA, PIA y el IIC son los tres indicadores cuyo valor idóneo alcanza el mayor número de participantes y, además, este buen número parece sostenerse con el transcurso de los foros, excepto en GD donde el número de participantes que alcanzan el valor idóneo del PIA disminuye en el F3 y se sostiene hasta el F5. 
Con respecto al último indicador, el IIR, Ilama la atención que únicamente el GB es el grupo en el que al menos cuatro o más participantes alcanzan siempre el valor idóneo, mientras que en el resto de los grupos este número fluctúa en cada foro.

Tabla 5. Número de participantes que alcanzan el valor idóneo de los indicadores en cada foro

\begin{tabular}{ccccccc}
\hline Grupo & Indicadores & $F 1$ & $F 2$ & $F 3$ & $F 4$ & $F 5$ \\
\hline GA & IIA & 5 & 6 & 6 & 6 & 6 \\
$(\mathrm{~N}=6)$ & PIA & 4 & 6 & 6 & 6 & 5 \\
& IIC & 6 & 5 & 6 & 6 & 6 \\
& IIR & 2 & 6 & 3 & 6 & 3 \\
GB & IIA & 6 & 6 & 6 & 6 & 6 \\
$(\mathrm{~N}=6)$ & PIA & 5 & 6 & 6 & 6 & 6 \\
& IIC & 6 & 6 & 6 & 5 & 4 \\
& IIR & 2 & 6 & 4 & 4 & 5 \\
GC & IIA & 3 & 6 & 6 & 6 & 6 \\
$(\mathrm{~N}=6)$ & $\mathrm{PIA}$ & 1 & 5 & 6 & 6 & 6 \\
& IIC & 6 & 6 & 6 & 6 & 4 \\
& IIR & 0 & 4 & 5 & 4 & 2 \\
GD & IIA & 3 & 5 & 6 & 6 & 6 \\
$(\mathrm{~N}=6)$ & $\mathrm{PIA}$ & 1 & 5 & 4 & 4 & 4 \\
& IIC & 5 & 6 & 6 & 6 & 6 \\
& IIR & 0 & 2 & 3 & 1 & 2 \\
\hline
\end{tabular}

En la tabla 6 se presenta el número de indicadores de IED que los participantes (P1, P2, P3, P4, P5 y P6 en cada grupo) cumplen en cada uno de los cinco foros (F1, F2, F3, F4 y F5), así como el número de veces que obtienen el perfil idóneo de actividad al cumplir con los cuatro indicadores de naturaleza estructural analizados. Las celdas sombreadas con gris corresponden a los foros en los que el participante alcanza el perfil idóneo para el ejercicio de la IE. 
Tabla 6. Número de indicadores de IE que los participantes cumplen en cada foro

\begin{tabular}{|c|c|c|c|c|c|c|c|}
\hline Grupo & $\begin{array}{c}\text { Foro } \\
\text { Participantes }\end{array}$ & $\mathrm{F} 1$ & F2 & F3 & F4 & F5 & $\begin{array}{l}\mathrm{N}^{\circ} \text { foros (F2-F5) } \\
\text { con todos los } \\
\text { indicadores }\end{array}$ \\
\hline \multirow{6}{*}{$\begin{array}{c}\mathrm{GA} \\
(\mathrm{N}=6)\end{array}$} & $\mathrm{P} 1$ & 1 & 4 & 3 & 4 & 2 & 2 \\
\hline & P2 & 4 & 3 & 4 & 4 & 4 & 3 \\
\hline & P3 & 4 & 4 & 4 & 4 & 3 & 3 \\
\hline & P4 & 3 & 4 & 4 & 4 & 4 & 4 \\
\hline & P5 & 2 & 4 & 3 & 4 & 3 & 2 \\
\hline & P6 & 3 & 4 & 3 & 4 & 4 & 3 \\
\hline
\end{tabular}

$N^{0}$ de participantes con todos los indicadores

\begin{tabular}{ll|l|l|l|l|l|l}
\multirow{3}{*}{$\mathrm{G}$} & $\mathrm{P} 1$ & 2 & 4 & 4 & 3 & 3 & 2 \\
\cline { 3 - 7 }$(\mathrm{NB}=6)$ & $\mathrm{P} 2$ & 3 & 4 & 4 & 4 & 3 & 3 \\
\cline { 2 - 7 } & $\mathrm{P} 3$ & 3 & 4 & 3 & 3 & 4 & 2 \\
\cline { 2 - 7 } & P4 & 3 & 4 & 4 & 4 & 4 & 4 \\
& P5 & 4 & 4 & 3 & 3 & 3 & 1 \\
\hline & P6 & 4 & 4 & 4 & 4 & 4 & 4 \\
\hline
\end{tabular}

$\mathrm{N}^{\circ}$ de participantes con todos los indicadores

\begin{tabular}{|c|c|c|c|c|c|c|c|}
\hline \multirow{6}{*}{$\begin{array}{c}\mathrm{GC} \\
(\mathrm{N}=6)\end{array}$} & P1 & 0 & 2 & 3 & 3 & 4 & 1 \\
\hline & P2 & 1 & 3 & 4 & 4 & 3 & 2 \\
\hline & P3 & 2 & 4 & 4 & 4 & 2 & 3 \\
\hline & P4 & 1 & 4 & 4 & 4 & 2 & 3 \\
\hline & P5 & 2 & 4 & 4 & 3 & 4 & 3 \\
\hline & P6 & 3 & 4 & 4 & 4 & 3 & 3 \\
\hline
\end{tabular}

$\mathrm{N}^{\circ}$ de participantes con todos los indicadores

\begin{tabular}{|c|c|c|c|c|c|c|c|}
\hline \multirow{6}{*}{$\begin{array}{c}\mathrm{GD} \\
(\mathrm{N}=6)\end{array}$} & $\mathrm{P} 1$ & 3 & 4 & 2 & 3 & 3 & 1 \\
\hline & P2 & 1 & 4 & 3 & 2 & 3 & 1 \\
\hline & P3 & 0 & 1 & 2 & 2 & 2 & 0 \\
\hline & P4 & 1 & 3 & 4 & 3 & 4 & 2 \\
\hline & P5 & 2 & 3 & 4 & 3 & 2 & 1 \\
\hline & P6 & 2 & 3 & 4 & 3 & 4 & 2 \\
\hline
\end{tabular}

$\mathrm{N}^{\circ}$ de participantes con todos los indicadores 
Como se aprecia en la tabla 6 , en términos generales, las mayores diferencias entre el número de indicadores que cumplen los participantes de los distintos grupos se sitúan principalmente entre el primer foro, en el que no reciben ningún tipo de información, y el resto de los foros, donde sí la reciben. En concreto, en el F1 aparecen dos participantes en los grupos GA y GB que cumplen con el perfil idóneo de actividad, es decir, que alcanzan el valor idóneo de los cuatro indicadores de naturaleza estructural; en cambio, en GC y GD no se observan participantes que cumplan con esta característica. Después, tras la primera entrega de información, en el F2 la gran mayoría de los participantes alcanza dicho perfil, excepto en GD en donde solamente aparecen dos participantes que cumplen con el valor idóneo de los cuatro indicadores. De manera similar, el número de participantes con perfil idóneo en el resto de los foros es siempre mayor que en el F1, menos en GD que presenta un foro (F4) en el que nuevamente ningún participante alcanza el valor idóneo de los cuatro indicadores.

Al contrastar los grupos, haciendo una lectura horizontal de la tabla 6 , se aprecia que en los foros en los que reciben información (F2-F5) el número de participantes que alcanza el perfil idóneo en tres o más foros es mayor en GA (cuatro participantes, P2, P3, P4 y P6), en GB (tres participantes, P2, P4 y P6) y en GC (cuatro participantes, P3, P4, P5 y P6), a diferencia de GD en el que no se observan participantes que cumplan con esta característica. Al hacer una lectura por foros, de manera vertical, también se observa que globalmente el GD presenta menos participantes con perfil idóneo, ya que ningún foro presenta más de tres participantes que alcancen el valor idóneo de los cuatro indicadores; por el contrario, GA, GB y GC se distinguen porque en cada foro en el que reciben información hay tres o más participantes con perfil idóneo, exceptuando el F5 del GC en donde únicamente aparecen dos participantes con esta característica.

\subsection{Evolución de las valoraciones sobre la utilidad de la información}

Los cuestionarios que cumplimentaron los estudiantes al finalizar los foros F2 a F5 exploraban dos aspectos concretos: el grado de utilidad de las informaciones recibidas para mejorar el proceso de colaboración y para mejorar la propia actividad individual. En la tabla 7 se muestra el total de puntuaciones de los miembros del grupo otorgadas a cada uno de estos aspectos en una escala Likert, donde 1 punto correspondía a muy poco útil y 4 a muy útil. Las celdas sombreadas corresponden a las valoraciones sobre la utilidad de la información para mejorar el proceso colaborativo; las celdas sin sombrear corresponden a las valoraciones sobre la utilidad de la información para mejorar la actividad individual. 
Tabla 7. Valoraciones sobre la utilidad de las informaciones para mejorar la participación en los foros siguientes

\begin{tabular}{lllll}
\hline & F2 & F3 & F4 & F5 \\
\hline \multirow{3}{*}{ GA } & 27 & 26 & 24 & 22 \\
& 26 & 24 & 24 & 21 \\
& 28 & 26 & 26 & 25 \\
GB & 25 & 24 & 24 & 25 \\
& 27 & 27 & 26 & 26 \\
GC & 27 & 26 & 26 & 27 \\
\hline
\end{tabular}

En términos generales, en los tres grupos que recibieron información se observa una disminución progresiva de las puntuaciones dadas por los participantes a cada uno de los ítems. Como se observa, las puntuaciones que los participantes conceden a la utilidad de la información son más altas cuando la reciben por primera vez (al inicio de F2) y en los foros siguientes estas puntuaciones suelen ser más bajas; este patrón se observa tanto en las puntuaciones de la utilidad de la información para mejorar el proceso de colaboración como la propia actividad de los participantes.

En el caso de los grupos GB y GC Ilama la atención el patrón que presentan las puntuaciones otorgadas a la utilidad de la información para mejorar la actividad individual (celdas sin sombrear), ya que a pesar de que estas puntuaciones bajan mínimamente en dos foros subsecuentes a la primera valoración, en los foros restantes estas puntuaciones son iguales a la que presenta el primer foro.

\section{Discusión y conclusiones}

Con el propósito de avanzar en el diseño de herramientas analíticas más ricas, que consideren la complejidad de los procesos de enseñanza y aprendizaje (Dawson y Siemens, 2014), en este artículo se ha recogido la propuesta de métrica de Coll y colaboradores (Coll, Bustos, y Engel, 2015; Coll, Engel, y Niño 2017) para adaptarla a la plataforma digital Blackboard. Lo anterior obedece ante el Ilamado de atención que Brown (2012) y Ferguson (2012) hacen con respecto a la urgencia de diseñar herramientas analíticas con un sustento teórico sobre el aprendizaje. Es así, como el modelo de IED y la métrica propuesta desde dicho modelo teórico, permiten identificar perfiles diferenciados de la actividad de los participantes en función de su mayor o menor proximidad a un perfil teóricamente idóneo para el ejercicio de la IE. Además, cabe mencionar que este estudio se ha realizado en una situación educativa real y natural desarrollada en el marco de una asignatura 
que complementa las actividades presenciales con un trabajo sostenido en línea, superando las limitaciones que presentan muchos de los trabajos en este ámbito que se realizan en situaciones artificiales.

Los resultados presentados permiten realizar una serie de conclusiones. En primer término, tal parece que la entrega de información asociada al modelo teórico de IED tiene como efecto que un mayor número de participantes de los grupos (GA, GB y GC) que reciben algún tipo de información alcancen el perfil idóneo de IE frente al grupo (GD) que no recibe información asociada al modelo de IED.

En segundo término, aunque el efecto es más notable en los tres grupos tras la primera entrega de información, este efecto va disminuyendo paulatinamente con el transcurso de los foros. Además, tal parece que el hecho de contar con un solo tipo de información, como en el caso de los grupos GA y GB, contribuye a que la mayoría de los participantes alcance el valor idóneo de los indicadores asociados el ejercicio de la influencia educativa, ya que en cada foro se observa que la mitad o más de la mitad de los participantes cumple con dicha característica. Cabe hacer notar que, según los resultados, tal parece que cuando los participantes cuentan con algún tipo de información asociada al modelo teórico de IED se favorece el establecimiento de relaciones recíprocas, especialmente cuando esta información se deriva del propio contenido de sus contribuciones.

En tercer término, las valoraciones que realizan los estudiantes sobre la utilidad de la información recibida son la primera vez más altas y, a medida que se suceden los foros, disminuyen progresivamente. No obstante, conviene hacer notar que las puntuaciones que permanecen ligeramente más estables son aquellas que los participantes de los grupos (GB y GC) que recibieron información derivada del análisis de contenido de sus contribuciones, lo que podría indicar que la información de naturaleza cualitativa es mejor valorada por los participantes que la información de naturaleza cuantitativa. De hecho, según las valoraciones realizadas por los participantes de los mismos grupos puede decirse que la información resulta mucho más útil para mejorar la actividad individual que la actividad grupal. En este sentido, también es posible que el haber entregado únicamente información individual haya influido en las buenas valoraciones que recibió este aspecto.

Hasta aquí, los resultados obtenidos en este trabajo de carácter exploratorio, avalan el interés de la propuesta; pero, también ponen de manifiesto la necesidad de ampliar y enriquecer la aproximación utilizada en este trabajo. Por una parte, se considera especialmente pertinente analizar más detalladamente las dinámicas particulares que establecen los participantes en el seno del grupo como posible vía para comprender el efecto de la infor- 
mación en las mismas. Para ello, puede ser particularmente útil incorporar un análisis del contenido de las contribuciones de los participantes, que permita complementar las informaciones obtenidas a partir del análisis estructural y definir perfiles más complejos o modalidades distintas de ejercicio de la IE, que den cuenta de las dinámicas particulares que se instauran en los grupos.

Por otra parte, también se considera necesario profundizar en las razones por las cuales la información no parece tener un efecto continuado en la actividad de los participantes. En este sentido, una posible vía de desarroIlo de futuras investigaciones sería estudiar el efecto que tiene proporcionar información tanto individual como grupal, cuando los miembros de los grupos Ilevan un tiempo colaborando y han establecido unas dinámicas propias de trabajo conjunto. El retomar la consideración anterior permitiría explorar, en futuros estudios, la incidencia de la información en la actividad individual y de grupo y su incidencia en el aprendizaje colaborativo más sistemáticamente.

\section{Referencias bibliográficas}

Brown, M. (2012). Learning Analytics: Moving from concept to practice. Louisville, CO: EDUCAUSE Learning Initiative, 7. Recuperado de https://bit.ly/2n6vJ2W

Bustos, A. (2011). Presencia docente distribuida, influencia educativa y construcción del conocimiento en entornos de enseñanza y aprendizaje basados en la comunicación asíncrona escrita. Tesis doctoral. Universidad de Barcelona. Recuperado de https:// bit.Iy/2IR7mF2

Bustos, A., Coll, C., y Engel, A. (2009). Presencia docente distribuida en redes asíncronas de aprendizaje. Definición teórica y perspectiva multimétodo para su estudio. En F. Díaz Barriga, G. Hernández, y M. Rigo (Eds.), Aprender y enseñar con TIC en educación superior: contribuciones del socioconstructivismo (pp. 97-128). México: Publicaciones Universidad Nacional Autónoma de México.

Coll, C. (2010). Enseñar y aprender, construir y compartir: procesos de aprendizaje y ayuda educativa. En C. Coll (Coord.), Desarrollo, aprendizaje y enseñanza en la Educación Secundaria (pp. 31-62). Barcelona: Graó

Coll, C., Bustos, A., y Engel, A. (2011). Perfiles de participación y presencia docente distribuida en redes asíncronas de aprendizaje: la articulación del análisis estructural y de contenido. Revista de Educación, 354, 657-688. Recuperado de https://bit. Iy/2US9mOK

Coll, C., Bustos, A. y Engel, A. (2015). La información sobre el ejercicio de la influencia educativa como medio para favorecer la participación y el aprendizaje en un foro en línea. Infancia y Aprendizaje, 38, 368-401. https://doi.org/10.1080/021037 $\underline{02.2015 .1016745}$

Coll, C., Bustos, A., Engel, A., de Gispert, I., y Rochera, M. J. (2013). Distributed Educational Influence and Computer-Supported Collaborative Learning. Digital Education Review, 24, 23-42. Recuperado de https://bit.ly/2J4ME3B 
Coll, C., Colomina, R., Onrubia, J., y Rochera, M. J. (1992). Actividad conjunta y habla: una aproximación a los mecanismos de influencia educativa. Infancia y Aprendizaje, 59-60, 189-132. Recuperado de https://bit.ly/2GTAQQ0

Coll, C., Engel, A., y Bustos, A. (2009). Distributed Teaching Presence and Participants' Activity Profiles: a theoretical approach to the structural analysis of Asynchronous Learning Networks. European Journal of Education, 44(4), 521-538. https://doi. org/10.1111/j.1465-3435.2009.01406.x

Coll, C., Engel, A., y Niño, S. (2017). La actividad de los participantes como fuente de información para promover la colaboración. Una analítica del aprendizaje basada en el modelo de Influencia Educativa Distribuida. RED Revista de Educación a Distancia, 53. https://doi.org/10.6018/red/53/2

Coll, C., Onrubia, J., y Mauri, T. (2008). Ayudar a aprender en contextos educativos: el ejercicio de la influencia educativa y el análisis de la enseñanza. Revista de Educación, 346, 33-70. Recuperado de https://bit.ly/2vvqUGe

Colomina, R., Onrubia, J., y Rochera, M. J. (2001). Interactividad, mecanismos de influencia educativa y construcción del conocimiento en el aula. En C. Coll, J. Palacios, y A. Marchesi (Comps.). Desarrollo Psicológico y educación. Vol. Il. Psicología de la Educación (pp. 415-435). Madrid: Alianza.

Dawson, S., y Siemens, G. (2014). Analytics to Literacies: The development of a learning analytics framework for multiliteracies assessment. The International Review of Research in Open and Distance Learning, 15(4), 284-305. Recuperado de https://bit.ly/2IQX2gi

Dehler, J., Bodemer, D., Buder, J., y Hesse, F. W. (2011). Guiding knowledge communication in CSCL via group knowledge awareness. Computers in Human Behavior, 27(3), 1068-1078. https://doi.org/10.1016/j.chb.2010.05.018

Dillenbourg, P., Järvelä, S., y Fischer, F. (2009). The evolution of research on computer-supported collaborative learning: from design to orchestration. En N. Balacheff, S. Ludvigsen, T. de Jong, T., A. Lazonder y S. Barnes (Eds.), Technology-Enhanced Learning. Principles and products (pp. 3-19). Springer, Dordrecht.

Dimitracopoulou, A. (2008). Computer based Interaction Analysis Supporting Self-regulation: Achievements and Prospects of an Emerging Research Direction. Technology, Instruction, Cognition and Learning, 6(4), 291-314. Recuperado de https://bit. ly/2DE547K

Durall, E., y Gros, B. (2014). Learning analytics as a metacognitive tool. In Proceedings of 6th International Conference on Computer Supported Education (CSEDU)(pp. 380-384). Recuperado de https://bit.ly/2XPw5ge

Engel, A., Coll, C., y Bustos, A. (2013). Distributed Teaching Presence and communicative patterns in asynchronous learning: Name versus reply networks. Computers $y$ Education, 60, 184-196. doi:10.1016/j.compedu.2012.06.011

Ferguson, R. (2012). The State Of Learning Analytics in 2012: A Review and Future Challenges. Technical Report KMI-12-01, Knowledge Media Institute, The Open University, UK. Recuperado de https://bit.ly/2WaE8ns

García, E., Romero, C., Ventura, S., y de Castro, C. (2011). A collaborative educational association rule mining tool. The Internet and Higher Education, 14(2), 77-88. https://doi. org/10.1016/j.iheduc.2010.07.006 
Gros, B. (2016). The design of smart educational environments. Smart Learning Environments, 3(15), 1-11. https://doi.org/10.1186/s40561-016-0039-x

Jeong, H., y Hmelo-Silver, C. E. (2016). Seven affordances of computer-supported collaborative learning: How to support collaborative learning? How can technologies help? Educational Psychologist, 51, 247-265. https://doi.org/10.1080/00461520.20 16.1158654

Kirschner, P. A., y Erkens, G. (2013). Toward a Framework for CSCL Research. Educational Psychologist, 48, 1-8. https://doi.org/10.1080/00461520.2012.750227

Siemens, G. y Long, P. (2011). Penetrating the Fog: Analytics in learning and Education. EDUCAUSE Review 46(5), 30-40. Recuperado de https://bit.ly/1xIMCED

Stahl, G., Koschmann, T., y Suthers, D. (2006). Computer-supported collaborative learning: An historical perspective. En R. K. Sawyer (Ed.), Cambridge handbook of the learning sciences (pp. 409-426). Cambridge, UK: Cambridge University Press. Recuperado de https://bit.ly/2vvrtzQ

Yin, R. K. (2006). Case Study methods. En J. L. Green, G. Camilli, y P. B. Elmore (Eds.), Handbook of Complementary Methods in Education Research (pp. 111-122). Mahwah, NJ: L. Erlbaum. 